\title{
Universiteit
}

Leiden

The Netherlands

\section{Osteoarthritis of the knee: Association between clinical features and MR imaging findings}

Kornaat, P.R.; Bloem, J.L.; Ceulemans, R.Y.T.; Riyazi, N.; Rosendaal, F.R.; Nelissen, R.G.; ... ; Kloppenburg, M.

\section{Citation}

Kornaat, P. R., Bloem, J. L., Ceulemans, R. Y. T., Riyazi, N., Rosendaal, F. R., Nelissen, R. G., ... Kloppenburg, M. (2006). Osteoarthritis of the knee: Association between clinical features and MR imaging findings. Radiology, 239(3), 811-817. Retrieved from https://hdl.handle.net/1887/5011

Version: $\quad$ Not Applicable (or Unknown)

License:

Downloaded from: https://hdl.handle.net/1887/5011

Note: To cite this publication please use the final published version (if applicable). 


\section{Osteoarthritis of the Knee:}

\section{Association between Clinical Features and MR Imaging Findings ${ }^{1}$}

Peter R. Kornaat, MD

Johan L. Bloem, MD

Ruth Y. T. Ceulemans, MD

Naghmeh Riyazi, MD

Frits R. Rosendaal, MD

Rob G. Nelissen, MD

Wayne 0. Carter, DVM, PhD

Marie-Pierre Hellio Le Graverand, MD

Margreet Kloppenburg, MD

${ }^{1}$ From the Departments of Radiology (P.R.K., J.L.B.), Rheumatology (N.R., M.K.), Clinical Epidemiology (M.K., F.R.R.), and Orthopaedic Surgery (R.G.N.), Leiden University Medical Center, Albinusdreef 2, NL-2333 ZA Leiden, the Netherlands; Department of Radiology, Northwestern Medical Faculty Foundation, Chicago, III (R.Y.T.C.); and Pfizer Groton, Groton, Conn (W.O.C., M.P.H.L.G.). Received February 15, 2005; revision requested April 13; revision received May 15; accepted June 13; final version accepted August 11. Supported by Pfizer Groton. Address correspondence to P.R.K. (e-mail: P.R.Kornaat @lumc.nl).

Purpose:

Materials and Methods:

Results:

Conclusion:
To prospectively evaluate the association between clinical features and structural abnormalities found at magnetic resonance $(\mathrm{MR})$ imaging in patients with osteoarthritis (OA) of the knee.

The study was approved by the institutional medical ethics review board. Written informed consent was obtained from each patient. MR images of the knee were obtained from 205 (42 [20\%] men, 163 [80\%] women; median age, 60 years; range, $43-77$ years) patients in whom symptomatic $\mathrm{OA}$ at multiple joint sites was diagnosed. MR images were analyzed for various abnormalities of OA. All patients were interviewed concerning pain and stiffness in the knee that was imaged. Odds ratios (ORs) with 99\% confidence intervals (CIs) were used to determine the association between the imaging findings and clinical features of OA.

A large joint effusion was associated with pain (OR, 9.99; 99\% CI: 1.28, 149) and stiffness (OR, 4.67; 99\% CI: 1.26 , 26.1). The presence of an osteophyte in the patellofemoral compartment (OR, 2.25; 99\% CI: 1.06, 4.77) was associated with pain. All other imaging findings, including focal or diffuse cartilaginous abnormalities, subchondral cysts, bone marrow edema, subluxation of the meniscus, meniscal tears, or Baker cysts, were not associated with symptoms.

Findings of this study indicate that only two associations exist between clinical symptoms and structural findings found on MR images in patients with OA of the knee.

(0) RSNA, 2006 
0 steoarthritis (OA) of the knee is a major cause of disability among the aging population of the industrialized world. A major hallmark of OA is loss of cartilage. The exact cause of knee pain in patients with OA remains enigmatic because hyaline cartilage does not contain pain fibers and, as such, cannot be the direct cause of pain in OA. Pain fibers are present in other structures in the knee, such as the joint capsule, periosteum, insertional sites of ligaments and muscles, outer third of the menisci, and, possibly, the synovium $(1,2)$, but their role is uncertain.

Radiographs remain the usual means for assessment of osteoarthritic changes in the knee and their association with clinical features, such as knee pain. The association between findings of $\mathrm{OA}$ on radiographs and clinical features, however, is poor (3). Magnetic resonance (MR) imaging allows another perspective of the structural abnormalities associated with $\mathrm{OA}$, and MR imaging findings have been associated with clinical features, which include knee pain. Reported findings include the association between knee pain and MR imaging findings, such as joint effusion and synovial thickening (4), bone marrow edema (5), osteophytes $(6,7)$, minimal cartilaginous lesions (8), alterations in volume of patellar cartilage (9), and periarticular lesions, which include bursitis and iliotibial band syndrome (10).

Controversy remains about the cause of knee pain in patients with OA. Findings in prior studies indicate that there is a poor association between knee pain and defects of cartilage; however, results in other studies indicate that there is an association between the two (9). Bone marrow edema was associated with clinical symptoms in the study of Felson et al (5), whereas this

\section{Advances in Knowledge}

- A large joint effusion is associated with pain and stiffness of the knee.

- The presence of an osteophyte in the patellofemoral compartment is associated with pain association was not found in the study of Link et al (8).

In most studies, knee pain was associated with only one structural abnormality or a limited number of them; furthermore, most of the studies have been based on small population samples (8). Thus, the purpose of our study was to prospectively evaluate the association between clinical features and structural abnormalities found at MR imaging in patients with $\mathrm{OA}$ of the knee.

\section{Materials and Methods}

Pfizer, Groton, Conn, provided financial support for this work; however, authors who are not employees of Pfizer had control of inclusion of all data and information that might present a conflict of interest for Pfizer employees.

\section{Patients}

Written informed consent was obtained from each patient. This study was approved by the medical ethics review board of Leiden University Medical Center, Leiden, the Netherlands.

The present prospective study is part of the ongoing Genetics, Osteoarthritis and Progression study (11). The primary goal of the Genetics, Osteoarthritis and Progression study is the identification of genetic susceptibility determinants to $\mathrm{OA}$ and disease progression in patients aged between 40 and 70 years who have generalized $\mathrm{OA}$. $\mathrm{Pa}$ tients who received a diagnosis determined by rheumatologists, orthopedic surgeons, and general practitioners were informed of the ongoing study by mail. Interested probands were mailed a questionnaire about demographic data, medical history, symptoms and signs of OA, and family history of OA. Subsequently, eligible probands were requested to introduce a sibling "with joint complaints" to the study. Between August 2000 and March 2003, 105 sibling pairs were included in the Genetics, Osteoarthritis and Progression-MR Imaging study. Probands and siblings were required to have symptomatic $\mathrm{OA}$ in at least two or more of the following joint sites: hands, spine (cervical or lumbar), knees, or hips. All sibling pairs who ful- filled the criteria were included (210 patients). In this study, 35\% (71 of 205) of the patients had symptomatic OA of the knee, and it was defined as pain or stiffness on most days of the prior month at study entry and the presence of osteophytes on radiographs. Radiographic evidence of $\mathrm{OA}$ of the knee, defined as a Kellgren and Lawrence score of greater than grade 1, was diagnosed in 47\% (97 of 205) of the patients (12). Because the purpose of the MR imaging study was to assess progression of $\mathrm{OA}$, no images were obtained of a knee that already had a maximum Kellgren and Lawrence score of grade 4 (12).

MR imaging of the knee was performed successfully in 205 (42 [20\%] men and 163 [80\%] women; median age, 60 years; range, 43-77 years) of 210 patients. The 210 patients consisted of 105 sibling pairs. Five patients were excluded. The remaining 205 patients consisted of 100 sibling pairs and five nonsiblings. Patients were excluded for the following reasons: claustrophobia in one, a large knee that would not fit into the knee coil in one, and inadequate image quality caused by motion artifacts in three.

\section{Clinical Assessment}

All patients were interviewed and underwent a physical examination by a rheumatologist (N.R.) with 3 years of experience. There was a single question

\section{Published online \\ 10.1148/radiol.2393050253 \\ Radiology 2006; 239:811-817 \\ Abbreviations: \\ $\mathrm{Cl}=$ confidence interval \\ $\mathrm{OA}=$ osteoarthritis \\ $\mathrm{OR}=$ odds ratio}

\section{Author contributions:}

Guarantor of integrity of entire study, P.R.K.; study concepts/study design or data acquisition or data analysis/ interpretation, all authors; manuscript drafting or manuscript revision for important intellectual content, all authors; manuscript final version approval, all authors; literature research, P.R.K., N.R.; clinical studies, P.R.K., J.L.B., R.Y.T.C., N.R., W.O.C., M.K.; statistical analysis, P.R.K., F.R.R., M.K.; and manuscript editing, P.R.K. J.L.B., F.R.R., R.G.N., W.O.C., M.P.H.L.G., M.K.

See Materials and Methods for pertinent disclosures. 
in the questionnaire in regard to these findings: Did you have knee pain and knee stiffness on most days of the prior month? A yes or no response to this question was requested. Some patients had OA of the hip, as well as OA of the knee. Referred pain from the ipsilateral hip may have been a confounder for pain in the knee; however, OA of the hip occurred in only 14 (7\%) of 205 patients.

\section{MR Image Acquisition}

Knees were imaged by using a transmitreceive four-channel knee coil and a 1.5-T superconducting magnet (Gyroscan Intera; Philips Medical Systems, Best, the Netherlands). Each examination consisted of the following: coronal intermediate-weighted (repetition time msec/echo time msec, 2200/20) and T2weighted (2200/80) dual spin-echo images (number of signals acquired, two; section thickness, $5 \mathrm{~mm}$; intersection gap, $0.5 \mathrm{~mm}$; field of view, $160 \mathrm{~mm}$; acquisition matrix, $205 \times 256$; and number of sections, 18), sagittal intermediate-weighted (2200/20) and T2weighted (2200/80) dual spin-echo images (number of signals acquired, two; section thickness, $4 \mathrm{~mm}$; intersection gap, $0.4 \mathrm{~mm}$; field of view, $160 \mathrm{~mm}$; acquisition matrix, $205 \times 256$; and number of sections, 20), sagittal threedimensional T1-weighted spoiled gradient-echo frequency-selective fat-suppressed images $(46 / 2.5$; one signal acquired; flip angle, $40^{\circ}$; section thickness, $3 \mathrm{~mm}$; section overlap, $1.5 \mathrm{~mm}$; no gap; field of view, $180 \mathrm{~mm}$; acquisition matrix, $205 \times 256$; and number of sections, 80), and transverse intermediate-weighted (2500/7.1) and T2-weighted (2500/40) turbo spin-echo fat-suppressed images (number of signals acquired, two; section thickness, $2 \mathrm{~mm}$; no gap; field of view, $180 \mathrm{~mm}$; acquisition matrix, $205 \times 256$; and number of sections, 62). Total acquisition time, which included the initial survey sequence, was $30 \mathrm{~min}$ utes.

\section{MR Image Interpretation}

All MR images were analyzed in consensus by three readers (P.R.K., R.Y.T.C., and J.L.B., who had 3, 15, and 25 years of experience, respectively) by using a comprehensive score form (13). During the assessment, the readers were blinded to radiographic results, patient symptoms, and patient age.

Cartilaginous defects, osteophytes, subchondral cysts, and bone marrow edema were assigned to one or more of the following anatomic locations: the crista patellae, medial or lateral patellar facets, the medial or lateral trochlear articular facets, the medial or lateral femoral condyles, and the medial or lateral tibial plateau. The medial and lateral menisci were reviewed for the presence of tears and extrusion from the joint line (subluxation). Joint effusion, synovitis, and Baker cysts were noted.

Cartilaginous defects were classified as diffuse or focal. Both coronal and sagittal spin-echo images and sagittal gradient-echo images were used to assess the tibiofemoral cartilage. Transverse turbo spin-echo images and sagittal gradient-echo and spin-echo images were used to assess the patellofemoral cartilage. The surface extent of a diffuse or focal cartilaginous defect was estimated with its maximal diameter and classified as follows: grade 0, absent; grade 1 , minimal $(<5 \mathrm{~mm})$; grade 2 , moderate $(5-10 \mathrm{~mm})$; or grade 3 , severe $(>10 \mathrm{~mm})$. The depth of a cartilaginous defect was classified by using a modification of the classification of Yulish et al (14): grade 0, absent (no abnormality in signal intensity or morphology); grade 1 , less than $50 \%$ reduction of thickness of cartilage; grade 2, 50\% or greater reduction of thickness of cartilage; or grade 3 , full-thickness or near full-thickness cartilaginous defect.

Osteophytes were defined as focal bony excrescences that were seen on transverse, sagittal, or coronal images and that extended from a cortical surface. The location of osteophytes was further specified as marginal, intercondylar, or central. A central osteophyte arose from the subchondral bone plate and was surrounded, but not necessarily covered, by articular cartilage. Osteophytes were classified with the following scale: grade 0 , absent; grade 1 , minimal $(<3 \mathrm{~mm})$; grade 2 , moderate
(3-5 mm); or grade 3 , severe $(>5 \mathrm{~mm})$. The size of the osteophyte was a measurement from the base to the tip (15). Each compartment (except the crista patellae) was screened for the possible presence of a marginal osteophyte, and only the medial and lateral femoral condyles were screened for the possible presence of an intercondylar osteophyte. Thus, a maximum of 10 marginal and intercondylar osteophytes combined could be counted. Each compartment was screened for the possible presence of a central osteophyte. Thus, a maximum of nine central osteophytes could be counted.

Subchondral cysts were characterized as well-defined foci of high signal intensity on $\mathrm{T} 2$-weighted images in the cancellous bone underlying the joint cartilage. Their greatest dimension was measured, and they were classified as follows: grade 0 , absent; grade 1 , minimal $(<3 \mathrm{~mm})$; grade 2 , moderate $(3-5$ $\mathrm{mm})$; or grade 3 , severe $(>5 \mathrm{~mm})$.

Bone marrow edema was characterized as an ill-defined area of increased signal intensity on T2-weighted gradient-echo images in the subchondral cancellous bone that extended away from the articular surface over a variable distance or that was in places where traction edema occurs (16). The lesions were classified as follows: grade 0 , absent; grade 1 , minimal (diameter of $<5$ $\mathrm{mm}$ ); grade 2 , moderate (diameter of 5 $\mathrm{mm}$ to $2 \mathrm{~cm}$ ); or grade 3 , severe (diameter of $>2 \mathrm{~cm}$ ).

A meniscal tear was defined as a region of intermediate signal intensity on intermediate-weighted images within the meniscus, which communicated with the superior or inferior surface or inner margin of the meniscus on more than one section.

Meniscal subluxation was defined as protrusion over the edge of the tibial plateau on coronal intermediate-weighted images and was classified as follows: grade 0 , absent; grade 1 , minimal (less than one-third width of the meniscus bulging); grade 2 , moderate (one- to twothirds of the meniscal width was involved); or grade 3 , severe (more than two-thirds of the meniscal width was involved). 
Presence of a knee joint effusion was evaluated on T2-weighted coronal, sagittal, and transverse images. A small physiologic sliver of synovial fluid was not recorded. A small effusion was present when a small amount of fluid distended one or two of the joint recesses, a moderate effusion was present when more than two joint recesses were partially distended, and a massive effusion was present when there was full distension of all the joint recesses. The lateral, medial, and suprapatellar joint recesses were evaluated.

A Baker cyst, or a distended bursa of the gastrocnemius muscle or a bursa of the semimembranosus muscle, was diagnosed when a circumscribed mass with intermediate signal intensity on intermediate-weighted spin-echo images and high signal intensity on T2-weighted dual spin-echo images was observed. This mass originated from the posteromedial tibiofemoral joint space, extended between the tendons of the medial head of the gastrocnemius and the semimembranosus muscles, and dis-

\section{Table 1}

Characteristics in 205 Patients with Symptomatic $0 A$ at Multiple Joints

\begin{tabular}{lc} 
Characteristic & Value \\
\hline Age $(\mathrm{y})$ & $60(43-77)$ \\
Weight $(\mathrm{kg})$ & $74(54-116)$ \\
Height $(\mathrm{cm})$ & $169(152-191)$ \\
Body mass index & $26(20-40)$ \\
Sex & \\
$\quad$ F & $163(80)$ \\
M & $42(20)$ \\
Knee pain & $121(59)$ \\
Knee stiffness & $97(47)$ \\
Knee OA* & $71(35)$ \\
Radiographically diagnosed & \\
knee OA $^{\dagger}$ & $97(47)$ \\
\hline
\end{tabular}

Note.-Values for age, weight, height, and body mass index are medians, and numbers in parentheses are ranges. Values for sex, knee pain, knee stiffness, knee $\mathrm{OA}$, and radiographically diagnosed knee $\mathrm{OA}$ are numbers of patients, and numbers in parentheses are percentages.

* Knee OA was defined as pain or stiffness on most days of the prior month, with osteophytes seen on radiographs.

${ }^{\dagger}$ Radiographically diagnosed knee OA was defined according to a Kellgren and Lawrence score of greater than 1 (12). sected caudally, cranially, or both. Bursal distension was classified as minimal, moderate, or severe.

\section{Statistical Analysis}

In the cross-sectional analyses, odds ratios (ORs) with 99\% confidence intervals (CIs) were used to show the association between the presence or absence of pain and stiffness and the presence or absence of the various osteoarthritic abnormalities. Since multiple tests were performed, a $P$ value of .01 or less was considered to indicate a significant difference. Logistic regression analysis was used to adjust for intrafamily effect and for the most important risk factors of OA: age, sex, and body mass index. Statistical software (Stata, version 7.0; Stata, College Station, Tex) was used for this purpose (P.R.K., M.K., F.R.R.).

\section{Results}

The overall characteristics of the patients are shown (Table 1). The frequency distribution of $\mathrm{OA}$ lesions in the population in this study showed that cartilaginous defects and osteophytes were the lesions seen most commonly on MR images of the knee (Tables 2, 3).

\section{Table 2}

\begin{tabular}{|c|c|}
\hline \multicolumn{2}{|c|}{$\begin{array}{l}\text { Frequency Distribution of } \\
\text { Abnormalities of OA Seen on MR } \\
\text { Images in } 205 \text { Patients }\end{array}$} \\
\hline Abnormalities & No. of Patients* \\
\hline Cartilaginous defects & $175(85)$ \\
\hline Diffuse & $167(81)$ \\
\hline Focal & 75 (37) \\
\hline Osteophytes & $174(85)$ \\
\hline Central osteophytes & $47(23)$ \\
\hline Subchondral cysts & $89(43)$ \\
\hline Bone marrow edema & $102(50)$ \\
\hline $\begin{array}{l}\text { Bone marrow edema } \\
\text { grades } 2 \text { and } 3\end{array}$ & $36(18)$ \\
\hline Meniscal tears & $138(67)$ \\
\hline $\begin{array}{l}\text { Subluxation of } \\
\text { meniscus }\end{array}$ & 74 (36) \\
\hline Effusion & $112(55)$ \\
\hline $\begin{array}{l}\text { Effusion of grades } 2 \\
\text { and } 3\end{array}$ & $15(7)$ \\
\hline Baker cysts & $96(47)$ \\
\hline
\end{tabular}

\section{Cartilaginous Defects}

No association was found between any of the cartilaginous defects and pain or stiffness (Table 4). We did not find an association between the subgroup of grade 2 cartilaginous defects and pain (OR, 1.88; 99\% CI: 0.50, 7.06; $P=.22$ ) or stiffness (OR, 2.06; 99\% CI: 0.57, 7.43; $P=.14)$

\section{Osteophytes}

The presence of an osteophyte in the entire knee was not associated with either of the two clinical features of pain and stiffness. The presence of an osteophyte in the patellofemoral compartment was associated with pain only (OR, 2.25; 99\% CI: 1.06, 4.77; $P=$ .005). No association between the grade of an osteophyte and knee pain (OR, 1.70; 99\% CI, 0.52, 5.50; $P=.25$ ) or stiffness (OR, 1.60; 99\% CI: 0.52 , 4.91; $P=.28$ ) was found. There was an association between the number of osteophytes (any grade) and pain when there were more than four osteophytes in the entire knee (OR, 2.80; 99\% CI: $1.28,6.12 ; P=.001)$. An association was found between central osteophytes and stiffness in the knee (OR, 2.25; 99\% CI: $0.93,5.41 ; P=.016)$; however, the $P$ value was not significant. No association between central osteophytes and knee pain was found (OR, 1.65; 99\% CI: 0.67, 4.10; $P=.15)$.

\section{Bone Marrow Edema}

Bone marrow edema was not associated with pain or stiffness; in particular, no association was found between the grade (grades 2 and 3 ) of bone marrow edema and knee pain (OR, 1.13; 99\% CI: $0.41,3.11 ; P=.76$ ) or stiffness (OR, 1.30; 99\% CI: 0.48, 3.55; $P=$ $.50)$. Sixty-four (53\%) of 121 patients with knee pain had bone marrow edema in the entire knee, and 38 (45\%) of 84 patients without knee, pain had bone marrow edema in the entire knee.

\section{Menisci, Effusion, and Baker Cyst}

No association was found between meniscal defects and pain or stiffness, and, in particular, no association was found between lesions of the medial (OR, 1.47; 99\% CI: $0.71,3.08 ; P=.44$ ) or 
lateral menisci (OR, 0.96; 99\% CI: 0.46, $2.00 ; P=.88)$ and knee pain.

An association was found between moderate and massive effusion (grades 2 and 3 ) and knee pain (OR, 9.99; 99\% CI: $1.28,149 ; P=.008)$ and between moderate to severe effusion (grades 2 and 3 ) and stiffness (OR, 4.67; 99\% CI: $1.26,26.1 ; P=.01)$ (Figure).

The presence of a Baker cyst was not associated with clinical findings. We did not find an association between severe (grades 2 and 3) Baker cysts and pain (OR, 1.90; 99\% CI: 0.56, 6.40; $P=$ $.17)$ and stiffness (OR, 1.88; 99\% CI: $0.61,5.76 ; P=.14)$.

ORs and CIs did not change essentially when we adjusted the data for intrafamily effect and for the most important risk factors of OA: age, sex, and body mass index.

\section{Discussion}

Although different grades and types of structural abnormalities in various anatomic compartments of the knee were comprehensively analyzed and were associated with pertinent clinical features, a remarkably small number of associations were found between the findings on MR images and knee pain or stiffness.

An association between osteophytes and knee pain was found only when an osteophyte was located in the patellofemoral compartment or when more than four (median) osteophytes were present anywhere in the knee. Boegard et al (7) reported an association between osteophytes at the inferior pole of the patella and knee pain. Boegard et al (6) also found an association between knee pain and osteophytes at the medial tibial plateau.

The frequency of central osteophytes in our study was 23\% (47 of 205 patients) and, in concordance with data in the literature (15), almost all central osteophytes (20 of 23 osteophytes) were associated with a full-thickness cartilaginous defect. An association between central osteophytes and knee stiffness was suggested $(P=.02)$, but central osteophytes were not associated with knee pain. These findings also

\begin{tabular}{|c|c|c|c|}
\hline \multicolumn{4}{|c|}{$\begin{array}{l}\text { Frequency Distribution of Abnormal Findings per Patient for Each Category of } \\
\text { Abnormality }\end{array}$} \\
\hline \multirow[b]{2}{*}{ Abnormality } & \multicolumn{3}{|c|}{ Findings* } \\
\hline & Median & Minimum & Maximum \\
\hline Cartilaginous defects & 3 & 0 & 18 \\
\hline Diffuse & 3 & 0 & 9 \\
\hline Focal & 0 & 0 & 9 \\
\hline Osteophytes & 4 & 0 & 10 \\
\hline Central osteophytes & 0 & 0 & 9 \\
\hline Subchondral cysts & 0 & 0 & 9 \\
\hline Bone marrow edema & 0 & 0 & 9 \\
\hline Meniscal tears & 1 & 0 & 6 \\
\hline Subluxation of meniscus & 0 & 0 & 2 \\
\hline Effusion & 0 & 0 & 1 \\
\hline Baker cysts & 0 & 0 & 1 \\
\hline
\end{tabular}

* The minimum and the maximum signify the minimum and maximum amounts of lesions that can be assigned a score per patient, respectively.

\section{Table 4}

Association between Basic MR Imaging Findings and Clinical Findings in 205 Patients

\begin{tabular}{|c|c|c|c|c|c|c|}
\hline \multirow[b]{3}{*}{ MR Imaging Findings } & \multicolumn{6}{|c|}{ Clinical Findings } \\
\hline & \multicolumn{3}{|c|}{ Pain } & \multicolumn{3}{|c|}{ Stiffness } \\
\hline & $\overline{P \text { Value }}$ & OR & $99 \% \mathrm{Cl}$ & $\overline{P \text { Value }}$ & $\mathrm{OR}$ & $99 \% \mathrm{Cl}$ \\
\hline Cartilaginous defects & .78 & 1.12 & $0.40,3.14$ & .45 & 1.35 & $0.48,3.82$ \\
\hline Focal & .83 & 1.07 & $0.50,2.28$ & .51 & 1.21 & $0.57,2.56$ \\
\hline Diffuse & .11 & 1.79 & $0.70,4.55$ & .19 & 1.61 & $0.62,4.20$ \\
\hline In patellofemoral compartment & .21 & 1.52 & $0.64,3.61$ & .35 & 1.37 & $0.57,3.26$ \\
\hline In tibiofemoral compartment & .20 & 1.47 & $0.68,3.17$ & .11 & 1.62 & $0.75,3.50$ \\
\hline Osteophytes & .91 & 1.05 & $0.38,2.91$ & .36 & 1.44 & $0.52,4.04$ \\
\hline Central osteophytes & .15 & 1.65 & $0.67,4.10$ & .02 & 2.25 & $0.93,5.41$ \\
\hline In patellofemoral compartment & .00 & 2.25 & $1.06,4.77^{\star}$ & .03 & 1.83 & $0.88,3.81$ \\
\hline In tibiofemoral compartment & .64 & 1.19 & $0.46,3.09$ & .97 & 0.99 & $0.38,2.56$ \\
\hline Subchondral cysts & .06 & 1.71 & $0.81,3.63$ & .44 & 1.25 & $0.60,2.59$ \\
\hline In patellofemoral compartment & .06 & 1.83 & $0.80,4.16$ & .92 & 1.03 & $0.47,2.25$ \\
\hline In tibiofemoral compartment & .72 & 1.14 & $0.44,2.92$ & .08 & 1.88 & $0.74,4.77$ \\
\hline Bone marrow edema & .28 & 1.36 & $0.65,2.84$ & .11 & 1.57 & $0.76,3.25$ \\
\hline In patellofemoral compartment & .35 & 1.31 & $0.62,2.79$ & .48 & 1.22 & $0.58,2.56$ \\
\hline In tibiofemoral compartment & .36 & 1.38 & $0.55,3.48$ & .08 & 1.83 & $0.75,4.47$ \\
\hline Meniscal tear & .44 & 1.26 & $0.58,2.74$ & .14 & 0.64 & $0.30,1.39$ \\
\hline Subluxation of meniscus & .92 & 1.03 & $0.48,2.21$ & .10 & 1.63 & $0.76,3.46$ \\
\hline Effusion of grades 2 and 3 & .01 & 9.99 & $1.13,149^{\star}$ & .01 & 4.67 & $1.11,26.14^{*}$ \\
\hline Baker cysts & .07 & 1.68 & $0.80,3.53$ & .32 & 1.32 & $0.64,2.73$ \\
\hline
\end{tabular}

${ }^{*} P \leq .01$.

were described in the study of Link et al (8) in which an association between central osteophytes and knee stiffness, but not knee pain, was found.

In our study, we did not find an association between the presence, severity, or frequency of bone marrow edema and pain or stiffness of the knee. These findings are supported by those in studies of Link et al (8) and Sowers et al (17) but are in contrast to results published by Felson et al (5). In the latter study, 
it was concluded that bone marrow edema on MR images was strongly associated with the presence of pain in OA of the knee. Felson et al reported findings in 401 patients with radiographically diagnosed OA. Seventy-eight percent of 351 patients with knee pain had bone marrow edema, whereas only $30 \%$ of 50 patients without knee pain had bone marrow edema. In our study, $64(53 \%)$ of 121 patients with knee pain had bone marrow edema in the entire knee, and 38 (45\%) of 84 patients without knee pain had bone marrow edema in the entire knee. These numbers show a clear disparity in prevalence of bone marrow edema in different populations. In the present study, patients with moderate (Kellgren and Lawrence score of $<4)$ OA and patients with no radiographically diagnosed OA of the knee but $\mathrm{OA}$ in the hands, spine, or hips were included, whereas in the study of Felson and colleagues, patients with more severe radiographically diagnosed $\mathrm{OA}$ (Kellgren and Lawrence score of 2-4) were included.

Another association found between structural abnormalities seen on MR images of the knee and clinical features was that between grade 2 and 3 (moderate and massive) joint effusion and knee pain or knee stiffness. In the literature,

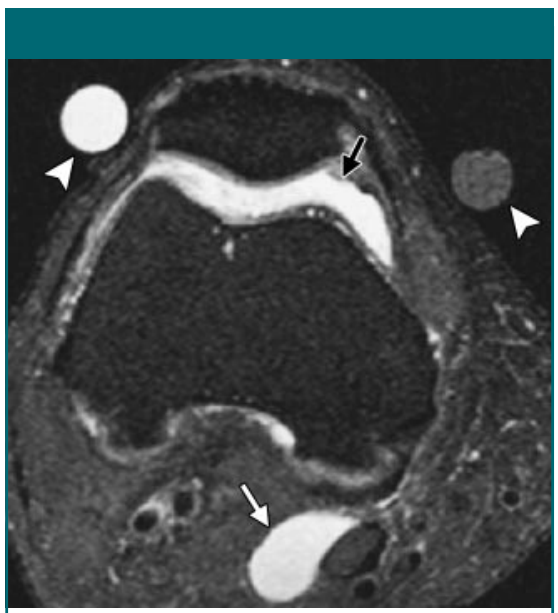

Transverse T2-weighted MR image (2500/40) shows moderate joint effusion (black arrow) associated with pain and stiffness of the knee. Note the presence of a Baker cyst (white arrow) and external markers (arrowheads). a controversy exists about the association between joint effusion and knee pain. Hill et al (4) found that moderate and severe effusions (grade 2 and 3 ) were substantially more common among patients with knee pain compared with those without who have OA of the knee. Link et al (8) reported no significant association between the presence or the amount of joint effusion and clinical features. They did, however, find a trend toward higher pain scores in patients with joint effusion. In some studies $(4,18,19)$, an explanation for the association between pain and joint effusion has been given in that the researchers suggested that capsular distention is the cause of knee pain.

No association was found between the number and grades of cartilaginous defects and clinical features. As anticipated, this lack of association conforms to data in the literature (8) because hyaline cartilage does not contain pain fibers, and, thus, cartilage cannot be the direct cause of pain in OA (1). In a study by Link et al (8), most symptoms were found in patients with grade IIa $(<50 \%$ loss of cartilage) cartilaginous lesions. They hypothesized that clinical symptoms are most substantial at the onset of OA, when cartilaginous defects occur. In that study, they found that when damage to cartilage progressed (grade 3 cartilaginous defects), a trend toward less pain and stiffness was shown, which may potentially be explained by the knee becoming more accustomed to the damage to the cartilage. These results could not be confirmed in the present study because no difference was found between grade 0 (no cartilaginous defects) and grade $2(>50 \%$ loss of cartilage, full-thickness loss of cartilage) cartilaginous defects.

No association was found between meniscal tears in patients with $\mathrm{OA}$ and clinical features. This finding is supported by findings in other studies in which the researchers reported that patients with or without a meniscal tear did not have significantly different levels of pain and disability $(8,20)$.

No association was found between the presence or grade of a Baker cyst and clinical features. This, too, has been reported, with popliteal cysts being as common in patients with knee pain as it is in those without (4).

In our study, we examined a broad spectrum of structural abnormalities found on MR images of the knee and the association between them and clinical features in a large customized study population. The study population was customized because it was based on data from siblings, and not all patients included had OA of the knee. The fact that patients were related according to family may have introduced an artifact. A logistic regression analysis, however, with robust standard errors that clustered on pairs accounted for this. A limitation of the study was the lack of a reference standard, such as arthroscopy. MR images, however, have been successfully used to evaluate cartilage and noncartilaginous joint structures (21-24), with accuracy values for imaging of cartilage of more than $90 \%$ $(25,26)$. Another limitation was that the recently improved imaging sequences for depiction of cartilage, such as steady-state free precession sequences, were not used (27-29). Thus, the presence of small focal cartilaginous defects might have been underestimated. A further limitation was that some patients had OA of the hip and of the knee. Although referred pain from the hip may have been a confounder, OA of the hip occurred in only 14 (7\%) of 205 patients. Last, we did not correct for the presence of intrasubstance degeneration of the meniscus when meniscal tears were associated with clinical features. Intrasubstance degeneration of the meniscus might be associated with knee pain (30).

In conclusion, findings in this study indicated that there were only two associations between structural abnormalities found on MR images and clinical features in patients with $\mathrm{OA}$ of the knee. Moderate and massive joint effusion was associated with both knee pain and knee stiffness. The presence of a patellofemoral osteophyte and the presence of more than four osteophytes in the entire knee were associated with knee pain only. Focal or diffuse cartilaginous abnormalities, subchondral cysts, 
bone marrow edema, subluxation of the meniscus, meniscal tears, or Baker cysts were not associated with pain and stiffness.

Acknowledgments: We acknowledge the support of the cooperating hospitals and referring rheumatologists, orthopedic surgeons, and general practitioners in our region.

\section{References}

1. Heppelmann B. Anatomy and histology of joint innervation. J Peripher Nerv Syst 1997; 2(1):5-16.

2. Dye SF, Vaupel GL, Dye CC. Conscious neurosensory mapping of the internal structures of the human knee without intraarticular anesthesia. Am J Sports Med 1998;26(6) :773777.

3. Lawrence JS, Bremner JM, Bier F. Osteoarthrosis: prevalence in the population and relationship between symptoms and $\mathrm{x}$-ray changes. Ann Rheum Dis 1966;25(1):1-24.

4. Hill CL, Gale DG, Chaisson CE, et al. Knee effusions, popliteal cysts, and synovial thickening: association with knee pain in osteoarthritis. J Rheumatol 2001;28(6):1330 1337.

5. Felson DT, Chaisson CE, Hill CL, et al. The association of bone marrow lesions with pain in knee osteoarthritis. Ann Intern Med 2001; 134(7):541-549.

6. Boegard T, Rudling O, Petersson IF, Jonsson K. Correlation between radiographically diagnosed osteophytes and magnetic resonance detected cartilage defects in the tibiofemoral joint. Ann Rheum Dis 1998;57(7): 401-407.

7. Boegard T, Rudling O, Petersson IF, Jonsson K. Correlation between radiographically diagnosed osteophytes and magnetic resonance detected cartilage defects in the patellofemoral joint. Ann Rheum Dis 1998;57(7): 395- 400 .

8. Link TM, Steinbach LS, Ghosh S, et al. Osteoarthritis: MR imaging findings in different stages of disease and correlation with clinical findings. Radiology 2003;226(2): 373-381.

9. Hunter DJ, March L, Sambrook PN. The association of cartilage volume with knee pain. Osteoarthritis Cartilage 2003;11(10): 725-729.

10. Hill CL, Gale DR, Chaisson CE, et al. Periarticular lesions detected on magnetic resonance imaging: prevalence in knees with and without symptoms. Arthritis Rheum 2003; 48(10): $2836-2844$.

11. Riyazi N, Meulenbelt I, Kroon HM, et al. Evidence for familial aggregation of hand, hip, and spine but not knee osteoarthritis in siblings with multiple joint involvement: the GARP study. Ann Rheum Dis 2005;64(3): $438-443$

12. Kellgren JH, Lawrence RC. Radiographic assessment of osteoarthritis. Ann Rheum Dis 1957;16:494-502.

13. Kornaat PR, Ceulemans RY, Kroon HM, et al. MRI assessment of knee osteoarthritis: Knee Osteoarthritis Scoring System (KOSS) inter-observer and intra-observer reproducibility of a compartment-based scoring system. Skeletal Radiol 2005;34(2):95-102.

14. Yulish BS, Montanez J, Goodfellow DB, Bryan PJ, Mulopulos GP, Modic MT. Chondromalacia patellae: assessment with MR imaging. Radiology 1987;164(3):763-766.

15. McCauley TR, Kornaat PR, Jee WH. Central osteophytes in the knee: prevalence and association with cartilage defects on MR imaging. AJR Am J Roentgenol 2001;176(2):359364

16. Mink JH, Deutsch AL. Occult cartilage and bone injuries of the knee: detection, classification, and assessment with MR imaging. Radiology 1989;170(3 pt 1):823-829.

17. Sowers MF, Hayes C, Jamadar D, et al. Magnetic resonance-detected subchondral bone marrow and cartilage defect characteristics associated with pain and x-ray-defined knee osteoarthritis. Osteoarthritis Cartilage 2003;11(6):387-393

18. Creamer P, Hunt M, Dieppe P. Pain mechanisms in osteoarthritis of the knee: effect of intraarticular anesthetic. J Rheumatol 1996; 23(6):1031-1036

19. Likar R, Schafer M, Paulak F, et al. Intraarticular morphine analgesia in chronic pain patients with osteoarthritis. Anesth Analg 1997;84(6):1313-1317
20. Bhattacharyya T, Gale D, Dewire P, et al The clinical importance of meniscal tears demonstrated by magnetic resonance imaging in osteoarthritis of the knee. J Bone Joint Surg Am 2003; 85-A(1):4-9.

21. Recht M, Bobic V, Burstein D, et al. Magnetic resonance imaging of articular cartilage. Clin Orthop Relat Res 2001;391(suppl):S379-S396

22. Peterfy CG. Scratching the surface: articular cartilage disorders in the knee. Magn Reson Imaging Clin N Am 2000;8(2):409-430.

23. Eckstein F, Reiser M, Englmeier KH, Putz R. In vivo morphometry and functional analysis of human articular cartilage with quantitative magnetic resonance imaging: from image to data, from data to theory. Anat Embryol (Berl) 2001;203(3):147-173

24. Vincken PW, ter Braak BP, van Erkel AR, et al. Effectiveness of MR imaging in selection of patients for arthroscopy of the knee. Radiology 2002;223(3):739-746.

25. Disler DG, McCauley TR, Kelman CG, et al. Fat-suppressed three-dimensional spoiled gradient-echo MR imaging of hyaline cartilage defects in the knee: comparison with standard MR imaging and arthroscopy. AJR Am J Roentgenol 1996;167(1):127-132.

26. Bredella MA, Tirman PF, Peterfy CG, et al Accuracy of T2-weighted fast spin-echo MP imaging with fat saturation in detecting cartilage defects in the knee: comparison with arthroscopy in 130 patients. AJR Am J Roentgenol 1999;172(4):1073-1080.

27. Gold GE, McCauley TR, Gray ML, Disler DG. What's new in cartilage? RadioGraphics 2003;23(5):1227-1242.

28. Hargreaves BA, Gold GE, Beaulieu CF, Vasanawala SS, Nishimura DG, Pauly JM Comparison of new sequences for high-resolution cartilage imaging. Magn Reson Med 2003;49(4):700-709.

29. Kornaat PR, Doornbos J, van der Molen AJ, et al. Magnetic resonance imaging of knee cartilage using a water selective balanced steady-state free precession sequence. J Magn Reson Imaging 2004;20(5):850 - 856.

30. Biedert RM. Treatment of intrasubstance meniscal lesions: a randomized prospective study of four different methods. Knee Surg Sports Traumatol Arthrosc 2000;8(2):104108. 